\title{
Real mixed Hodge structures
}

\author{
Mikhail Kapranov
}

\begin{abstract}
We identify the category of real mixed Hodge structures with the category of vector bundles with connections (not necessarily flat) on $\mathbb{C}$, equivariant with respect to $\mathbb{C}^{*}$. Here $\mathbb{C}$ is the complex plane considered as a 2-dimensional real manifold, and $\mathbb{C}^{*}$ is the multiplicative group of complex numbers considered as a real group.
\end{abstract}

Mathematics Subject Classification (2010). Primary 14C30; Secondary 58A14.

Keywords. Hodge structure, equivariant connection, twistor transform.

\section{Introduction}

Let $\mathrm{MHS}_{\mathbb{R}}$ be the category of real mixed Hodge structures [6], [7]. Thus, an object of $\mathrm{MHS}_{\mathbb{R}}$ consists of a finite-dimensional $\mathbb{R}$-vector space $V$, equipped with an increasing filtration $W_{\bullet}$ and a decreasing filtration $F^{\bullet}$ on $V_{\mathbb{C}}=V \otimes_{\mathbb{R}} \mathbb{C}$. These filtrations are required to satisfy the following condition:

$$
\operatorname{gr}_{F}^{p} \operatorname{gr}_{F}^{q} \operatorname{gr}_{n}^{W}\left(V_{\mathbb{C}}\right)=0 \text { for } n \neq p+q
$$

Here $\bar{F}^{\bullet}$ is the filtration obtained from $F^{\bullet}$ by complex conjugation, and we denote the filtration induced by $W_{\bullet}$ on $V_{\mathbb{C}}$ by the same symbol $W_{\bullet}$.

It is known that $\mathrm{MHS}_{\mathbb{R}}$ is an abelian tensor category. The goal of this note is to give a "gauge-theoretic" description of $\mathrm{MHS}_{\mathbb{R}}$.

Consider $\mathbb{C}$, the set of complex numbers, as a 2-dimensional real algebraic variety (i.e., an algebraic variety over $\mathbb{R}$ ), and the multiplicative group $\mathbb{C}^{*}$ as a 2-dimensional real algebraic group acting on this variety. In more formal algebro-geometric terms, we are applying the Weil restriction functor $R_{\mathbb{C} / \mathbb{R}}$ from $\mathbb{C}$ to $\mathbb{R}$ :

$$
\mathbb{C}=R_{\mathbb{C} / \mathbb{R}}\left(\mathbb{A}^{1}\right)=\mathbb{A}_{\mathbb{R}}^{2}, \quad \mathbb{C}^{*}=S:=R_{\mathbb{C} / \mathbb{R}}\left(\mathbb{G}_{m}\right),
$$

see [6], (2.1.2). Let $\operatorname{Bun}_{\nabla}\left(\mathbb{C} ; \mathbb{C}^{*}\right)$ be the category of (real) algebraic vector bundles on $\mathbb{C}$, equivariant with respect to the $\mathbb{C}^{*}$-action and equipped with an invariant (real algebraic) connection. Note that the connections are not assumed flat. 
Theorem 0.3. There exists an equivalence of abelian tensor categories

$$
h: \operatorname{Bun}_{\nabla}\left(\mathbb{C} ; \mathbb{C}^{*}\right) \rightarrow \mathrm{MHS}_{\mathbb{R}}
$$

with the following properties:

(a) If $(E, \nabla)$ is an object of $\operatorname{Bun}_{\nabla}\left(\mathbb{C} ; \mathbb{C}^{*}\right)$ and $\left(V, W_{\bullet}, F^{\bullet}, \bar{F}^{\bullet}\right)=h(E, \nabla)$, then the space $\mathrm{gr}^{W}(V)$ is canonically identified with $E_{0}$, the fiber of $E$ at 0 .

(b) In the situation of (a), the vector space $V$ is identified with the space of covariantly constant sections

$$
V=H_{\nabla}^{0}(\{t \mid \operatorname{Re}(t)=-1 / 2\}, E) .
$$

(c) $\nabla$ is flat if and only if $V$ is split, i.e., isomorphic to a direct sum of pure Hodge structures.

(d) The functor of "absolute cohomology"

$$
R \Gamma_{\text {Hod }}: \text { MHS } \rightarrow \operatorname{Vect}_{\mathbb{R}}, \quad V \mapsto R \operatorname{Hom}_{\mathrm{MHS}}(\mathbb{R}(0), V),
$$

of Beilinson [1] is identified with the invariant part of the "de Rham sequence" functor

$$
(E, \nabla) \mapsto \Gamma\left(\mathbb{C},\left\{E \stackrel{\nabla}{\rightarrow} E \otimes \Omega_{\mathbb{C}}^{1}\right\}\right)^{\mathbb{C}^{*}} .
$$

Here $\Omega_{\mathbb{C}}^{1}$ is the sheaf of real 1-forms on $\mathbb{C}$ as a real 2-manifold.

Remarks 0.4. (a) This gives a geometric interpretation of the pro-unipotent group $\mathcal{U}$ which, according to Deligne [7], governs mixed Hodge structures. Indeed, $\mathfrak{U}$ is realized as (the pro-algebraic completion of) the group of piecewise smooth loops in $\mathbb{C}$ considered up to reparametrization and cancellation (with the group operation being the composition of loops). This group of loops acts in any bundle with connection via the holonomy. See Section 2.7 below for a discussion at the Lie algebra level.

(b) Theorem 0.3 bears interesting similarities with the results of Connes and Marcolli [4]. They considered a certain tensor category of "equisingular" families of connections and identified it with the category of representations of a pro-unipotent group $U^{*}$. This group contains $\mathbb{G}_{m}$ and the commutant $\left[\mathcal{U}^{*}, \mathcal{U}^{*}\right]$ is free on generators of $\mathbb{G}_{m}$-weights $1,2,3, \ldots$ On the other hand, our results show that the category $\operatorname{Bun}_{\nabla}\left(\mathbb{C}, \mathbb{C}^{*}\right)$ is equivalent to the category of representations of the group $\mathfrak{U}$ of Deligne. This group contains $\mathbb{C}^{*}=S$, and $[\mathfrak{U}, \mathfrak{U}]$ is free on generators $z_{p, q}, p, q \geq 1$, of bi-weight $p, q$. So the relation between $\mathcal{U}$ and $\mathcal{U}^{*}$ is qualitatively the same as the relation between all mixed Hodge structures and iterated extensions of Tate structures. However, the two categories of connections leading to these groups are quite different and a direct relation of [4] with Hodge structures is not known.

(c) The group $\mathbb{C}^{*}$ does not act freely on $\mathbb{C}$ because of the fixed point at 0 , so from the topological point of view the quotient $\mathbb{C} / \mathbb{C}^{*}$ is ill behaved and should be replaced by the quotient stack $\mathbb{C} / / \mathbb{C}^{*}$. This stack can be seen as a local archimedean analog of 
the "adele class space" $A / / k^{*}$ of Connes [3]. Here $k$ is a global field, and $A$ is its ring of adeles. Quotients similar to $\mathbb{C} / / \mathbb{C}^{*}$ were also considered by Laumon [11] from the point of view of D-modules and constructible sheaves. Categories of equivariant connections on other pre-homogeneous vector spaces can provide interesting analogs of the category of mixed Hodge structures.

The proof of Theorem 0.3 will be given in $\S 1$. It is based on a version of the Radon-Penrose transform which takes equivariant bundles with connections on (the complexification of) $\mathbb{C}$ into equivariant algebraic vector bundles on a punctured projective plane. The latter bundles can be realized as Rees bundles of mixed Hodge structures, following the work of Penacchio [13], [14].

In $\$ 2$ we will relate our description with the description of Deligne [7] using the "Hodge monodromy operator". We also give an interpretation of Deligne's Hodge group $(5$ and its Lie algebra.

I am deeply grateful to A. B. Goncharov who explained to me some basics of mixed Hodge structures. He also informed me about his work [8] (then in progress). Theorem 0.3 turned out to be very closely related to the approach of [8]. In particular, the "twistor line" of [8] is naturally identified with the real line $\operatorname{Re}(t)=-1 / 2$ from Theorem 0.3 (c). See Remark 2.5 .2 below for more details. I would also like to thank F. Loeser for pointing out the work [11]. This work was partially supported by an NSF grant.

\section{Construction of the equivalence}

1.1. Complex version. We will deduce Theorem 0.3 from its complex version. Recall that a complex mixed Hodge structure is a datum consisting of a finite-dimensional complex vector space $V$ with an increasing filtration $W$ and two decreasing filtrations $F^{\prime}, F^{\prime \prime}$ which satisfy the condition

$$
\operatorname{gr}_{F^{\prime}}^{p} \operatorname{gr}_{F^{\prime \prime}}^{q} \operatorname{gr}_{n}^{W}(V)=0 \text { for } n \neq p+q
$$

similar to (0.1). Another name for such an object is "a triple of opposite filtrations" [6]. We denote by $\mathrm{MHS}_{\mathbb{C}}$ the category of complex mixed Hodge structures. Like $\mathrm{MHS}_{\mathbb{R}}$, it is an abelian tensor category.

For a complex vector space $V$ we denote $\sigma(V)$ the complex conjugate space. It consists of symbols $\sigma(v), v \in V$, with the operations

$$
\sigma(v)+\sigma\left(v^{\prime}\right)=\sigma\left(v+v^{\prime}\right), \quad \lambda \sigma(v)=\sigma(\bar{\lambda} v), \quad \lambda \in \mathbb{C} .
$$

Thus we have an antilinear isomorphism

$$
\sigma: V \rightarrow \sigma(V)
$$


Given a complex mixed Hodge structure $\left(V, W_{\bullet}, F^{{ }^{\bullet}}, F^{\prime \prime \bullet}\right)$, its complex conjugate structure is defined by

$$
\sigma\left(V, W_{\bullet}, F^{\prime \bullet}, F^{\prime \prime \bullet}\right)=\left(\sigma(V), \sigma\left(W_{\bullet}\right), \sigma\left(F^{\prime \prime \bullet}\right), \sigma\left(F^{\prime \bullet}\right)\right)
$$

This defines an action of the Galois group

$$
\Gamma=\operatorname{Gal}(\mathbb{C} / \mathbb{R})=\{1, \sigma\}
$$

on the category $\mathrm{MHS}_{\mathbb{C}}$, and $\mathrm{MHS}_{\mathbb{R}}$ consists of $\Gamma$-equivariant objects in $M H S_{\mathbb{C}}$. So we describe $\mathrm{MHS}_{\mathbb{C}}$ in a way compatible with the $\Gamma$-action.

Consider the affine plane $\mathbb{A}^{2}=\operatorname{Spec} \mathbb{C}\left[t_{1}, t_{2}\right]$ over $\mathbb{C}$ with the standard action of the torus $\mathbb{G}_{m}^{2}$. Let $\operatorname{Bun}_{\nabla}\left(\mathbb{A}^{2} ; \mathbb{G}_{m}^{2}\right)$ be the category of complex algebraic vector bundles on $\mathbb{A}^{2}$ which are equivariant with respect to the $\mathbb{G}_{m}^{2}$-action and equipped with an equivariant (complex algebraic) connection. There is a $\Gamma$-action on $\operatorname{Bun}_{\nabla}\left(\mathbb{A}^{2} ; \mathbb{G}_{m}^{2}\right)$ induced by the action on $\mathbb{A}^{2}$ given by

$$
\sigma\left(t_{1}, t_{2}\right)=\left(\bar{t}_{2}, \bar{t}_{1}\right)
$$

and by a similar action on $\mathbb{G}_{m}^{2}$. The following then implies Theorem $0.3(\mathrm{a})$.

Theorem 1.1.4. $\mathrm{MHS}_{\mathbb{C}}$ is equivalent to $\operatorname{Bun}_{\nabla}\left(\mathbb{A}^{2} ; \mathbb{G}_{m}^{2}\right)$ as a tensor category with $\Gamma$-action.

The proof will be given in Section 1.4 below.

1.2. The Radon-Penrose transform of equivariant connections. Let us compactify $\mathbb{A}^{2}=\operatorname{Spec} \mathbb{C}\left[t_{1}, t_{2}\right]$ to $\mathbb{P}^{2}=\operatorname{Proj} \mathbb{C}\left[u_{0}, u_{1}, u_{2}\right]$, so $\mathbb{A}^{2}$ is given by $u_{0} \neq 0$, and

$$
\mathbb{P}^{2}=\mathbb{A}^{2} \cup \mathbb{P}_{\infty}^{1}, \quad \mathbb{P}_{\infty}^{1}=\operatorname{Proj} \mathbb{C}\left[t_{1}, t_{2}\right], \quad t_{i}=u_{i} / u_{0}
$$

Let $\breve{\mathbb{P}}^{2}$ be the dual projective plane of lines in $\mathbb{P}^{2}$, so $\breve{\mathbb{P}}^{2}=\operatorname{Proj} \mathbb{C}\left[v_{0}, v_{1}, v_{2}\right]$, where $\left(v_{i}\right)$ are the dual coordinates. As usual, points in $\mathbb{P}^{2}$ give lines in $\mathbb{\mathbb { P }}^{2}$ : for a point $x \in \mathbb{P}^{2}$ we denote $\lambda_{x}$ the set of lines in $\mathbb{P}^{2}$ through $x$. Now, lines in $\mathbb{A}^{2}$ form

$$
\check{\mathbb{P}}_{0}^{2}=\check{\mathbb{P}}^{2}-\{[1: 0: 0]\},
$$

so we have the incidence diagrams

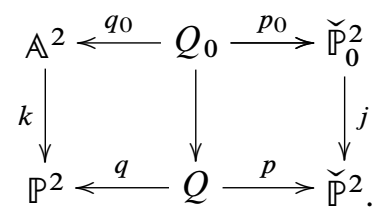


Proposition 1.2.3. Let $(E, \nabla) \in \operatorname{Bun}_{\nabla}\left(\mathbb{A}^{2} ; \mathbb{G}_{m}^{2}\right)$ and $r=\operatorname{rk}(E)$. Consider the sheaf of $\mathcal{O}$-modules on $\breve{\mathbb{P}}_{0}^{2}$,

$$
\mathcal{E}^{0}=p_{0 *}^{\nabla}\left(q_{0}^{*} E\right),
$$

where $p_{0 *}^{\nabla}$ is the subsheaf in the direct image $p_{0 *}$ consisting of sections covariantly constant along the fibers of $p_{0}$. Then $\mathcal{E}^{0}$ is locally free of rank $r$, so it is an algebraic vector bundle on $\mathbb{\mathbb { P }}_{0}^{2}$.

We call $\&^{0}$ the Radon-Penrose transform of $(E, \nabla)$, following [12], Ch. 2, §2.

Proof. The variety $\breve{\mathbb{P}}_{0}^{2}$ is covered by two open charts isomorphic to $\mathbb{A}^{2}$. One of them, $U=\operatorname{Spec} \mathbb{C}[a, b]$, parametrizes lines of the form

$$
t_{2}=a t_{1}+b,
$$

and the other one is defined similarly, with the roles of $t_{1}, t_{2}$ interchanged. So we will prove that $\left.\mathcal{E}^{0}\right|_{U}$ is a free $\mathcal{O}_{U}$-module of rank $r$.

Let $V$ be the space of $\mathbb{G}_{m}^{2}$-invariant sections of $E$ over $\mathbb{G}_{m}^{2}$, so $\operatorname{dim}(V)=r$. Over $\mathbb{G}_{m}^{2}$, we have a trivialization $E \simeq \mathcal{O}_{\mathbb{G}_{m}^{2}} \otimes V$. With respect to this trivialization, the equivariant connection $\nabla$ has the form

$$
\nabla=d+B_{1} d \log t_{1}+B_{2} d \log t_{2}, \quad B_{1}, B_{2} \in \operatorname{End}(V) .
$$

Now eq. (1.2.4) identifies $p_{0}^{-1}(U)$ with $U \times \mathbb{A}^{1}$, where $\mathbb{A}^{1}=\operatorname{Spec} \mathbb{C}\left[t_{1}\right]$. Denote by $\widetilde{E}$ the algebraic vector bundle on $U \times \mathbb{A}^{1}$ corresponding to the bundle $\left.q_{0}^{*}(E)\right|_{p_{0}^{-1}(U)}$ under this identification. Then $\nabla$ induces a relative connection in $\widetilde{E}$ along the fibers of the projection $U \times \mathbb{A}^{1} \rightarrow U$. Denote this relative connection by $D$. Substituting (1.2.4) into (1.2.5) and using the above trivialization of $E$ on $\mathbb{G}_{m}^{2}$, we find a trivialization of $\widetilde{E}$ near $U \times\{\infty\} \subset U \times \mathbb{P}^{1}$ such that the connection matrix of $D$ in this trivialization has at most first order pole near $t_{1}=\infty$.

This means that for each choice of numerical values $a, b \in \mathbb{C}$ we then have a nonsingular connection in an algebraic vector bundle $\widetilde{E}_{a, b}=\left.E\right|_{\left\{t_{2}=a t_{1}+b\right\}}$ on $\mathbb{A}^{1}$, having a regular singularity at infinity; see [5], Theorem 1.1.2(i). As such, it has a fundamental solution whose matrix elements are regular functions (polynomials) in $t_{1}$; see [5], Theorem 1.1.9. As $a, b$ vary, the coefficients of these polynomials are regular functions in $a, b$ since they are found by the standard recursive formulas. This implies that the relative connection $D$ in $\widetilde{E}$ is algebraically trivial (isomorphic to a pullback of a bundle on $U$, with trivial relative connection), and so the sheaf of covariantly constant sections is a free $\mathcal{O}_{U}$-module of rank $r$.

The action of the torus $\mathbb{G}_{m}^{2}$ on $\mathbb{A}^{2}$ by dilations extends to $\mathbb{P}^{2}$ and, by duality, to $\check{\mathbb{P}}^{2}$. Both $\mathbb{P}^{2}$ and $\check{\mathbb{P}}^{2}$ are toric varieties with respect to this action, and $\breve{\mathbb{P}}_{0}^{2}$ is a torus invariant open set. By construction, the vector bundle $\mathcal{E}^{0}$ on $\check{\mathbb{P}}_{0}^{2}$ is $\mathbb{G}_{m}^{2}$-equivariant. 

image

Note further that the embedding $j: \check{\mathbb{P}}_{0}^{2} \rightarrow \check{\mathbb{P}}^{2}$ misses just one point, so the direct

$$
\mathcal{E}=j_{*} \varepsilon^{0}
$$

is a reflexive coherent sheaf on the surface $\breve{\mathbb{P}}^{2}$ and thus a vector bundle. This bundle is still equivariant with respect to $\mathbb{G}_{m}^{2}$.

We denote by $\mathcal{Q}$ the category of vector bundles $\mathcal{E}$ on $\breve{\mathbb{P}}^{2}$ equivariant under $\mathbb{G}_{m}^{2}$ and trivial on each line $\lambda \subset \mathbb{P}^{2}$ except perhaps the lines corresponding to points of $\mathbb{P}_{\infty}^{1} \subset \mathbb{P}^{2}$. We have a natural structure of a tensor category on $\mathbb{Q}$ given by tensor product of vector bundles.

Theorem 1.2.7. The correspondence $(E, \nabla) \mapsto \mathcal{E}$ establishes an equivalence of tensor categories

$$
\operatorname{Bun}_{\nabla}\left(\mathbb{A}^{2} ; \mathbb{G}_{m}^{2}\right) \rightarrow \mathcal{Q}
$$

Proof. We already showed how to construct $\mathcal{E}$ from $(E, \nabla)$ and that $\mathcal{E}$ is equivariant. Note that $\mathcal{Q}$ is equivalent, via (1.2.6), to the category $\mathcal{Q}_{0}$ of equivariant bundles on $\breve{\mathbb{P}}_{0}^{2}$ trivial on all the lines contained in $\breve{\mathbb{P}}_{0}^{2}$. Indeed, these are precisely the lines not corresponding to the points of $\mathbb{P}_{\infty}^{1}$. Now, the equivalence of $\operatorname{Bun}_{\nabla}\left(\mathbb{A}^{2} ; \mathbb{G}_{m}^{2}\right)$ with $\mathcal{P}_{0}$ is an equivariant version of a particular case of the general fact about Radon-Penrose transforms ([12], Ch. 2, §2, Theorem 2.3). Indeed, the first line in (1.2.2) is a particular case of a double fibration considered in [12], Ch. 2, Sect. 2.1. The cited theorem establishes an equivalence between holomorphic bundles on $\breve{\mathbb{P}}_{0}^{2}$ trivial on all the lines and holomorphic bundles with connections on $\mathbb{A}^{2}$ but in our equivariant situation we can restrict to algebraic bundles on both sides, in virtue of Proposition 1.2.3. Let us just explain why $\mathcal{E}$ is trivial on each line contained in $\breve{\mathbb{P}}_{0}^{2}$, i.e., any line $\lambda$ of the form $\lambda_{x}, x \in \mathbb{A}^{2}$. Indeed, if $l \subset \mathbb{A}^{2}$ is a line through $x$, then the restriction of covariantly constant sections to $x$ gives an isomorphism

$$
\mathcal{E}_{l}=H_{\nabla}^{0}(l, E) \stackrel{\simeq}{\longrightarrow} E_{x},
$$

so

$$
\left.\mathcal{E}\right|_{\lambda}=\mathcal{O}_{\lambda} \otimes E_{x}
$$

is trivial.

1.3. Rees bundles and the work of Penacchio. We now relate Theorem 1.2.7 with the description of $\mathrm{MHS}_{\mathbb{C}}$ given by Penacchio [13], [14]. Denote by

$$
\operatorname{Rs}\left(V, W_{\bullet}, F^{\prime \bullet}, F^{\prime \prime \bullet}\right)=\bigoplus_{i, j, k \in \mathbb{Z}}\left(W_{i} \cap F^{\prime-j} \cap F^{\prime \prime-k}\right)(V) v_{0}^{i} v_{1}^{j} v_{2}^{k} \subset \mathbb{C}\left[v_{0}^{ \pm 1}, v_{1}^{ \pm 1}, v_{2}^{ \pm 1}\right]
$$

the Rees module over $\mathbb{C}\left[v_{0}, v_{1}, v_{2}\right]$ corresponding to the 3 -graded vector space $\left(V, W_{\bullet}, F^{\prime \bullet}, F^{\prime \prime}\right)$. Here the minus signs before $j$ and $k$ correspond to converting the decreasing filtrations $F^{\prime}, F^{\prime \prime}$ into increasing ones. Both $\mathbb{C}\left[v_{0}, v_{1}, v_{2}\right]$ and 
$\operatorname{Rs}\left(V, W_{\bullet}, F^{\prime}, F^{\prime \prime}\right)$ have compatible $\mathbb{Z}^{3}$-gradings which translate to a $\mathbb{G}_{m}^{3}$-action on $\overleftarrow{\AA}^{3}=\operatorname{Spec} \mathbb{C}\left[v_{0}, v_{1}, v_{2}\right]$ and into an equivariant structure of the coherent sheaf on $\widetilde{\AA}^{3}$ corresponding to $\operatorname{Rs}\left(V, W_{\bullet}, F^{\prime \bullet}, F^{\prime \prime \bullet}\right)$. If we consider the gradings by total degree, we can form the projective plane $\breve{\mathbb{P}}^{2}=\operatorname{Proj} \mathbb{C}\left[v_{0}, v_{1}, v_{2}\right]$ which we identify with the $\breve{\mathbb{P}}^{2}$ from Section 1.2, and a coherent sheaf on $\breve{\mathbb{P}}^{2}$ corresponding to the graded module $\operatorname{Rs}\left(V, W_{\bullet}, F^{\prime \bullet}, F^{\prime \prime \bullet}\right)$. We denote this sheaf $\mathbb{P R s}\left(V, W_{\bullet}, F^{\prime \bullet}, F^{\prime \prime \bullet}\right)$ or simply by $\mathcal{E}$. The quotient torus

$$
\mathbb{G}_{m}^{2}=\mathbb{G}_{m}^{3} / \mathbb{G}_{m}
$$

(quotient by the diagonal embedding) is identified with the $\mathbb{G}_{m}^{2}$ acting on $\breve{\mathbb{P}}^{2}$, and $\mathcal{E}$ is equivariant. Further, it is known that Rees modules are reflexive, so $\mathbb{E}$ is a $\mathbb{G}_{m}^{2}$ equivariant vector bundle on $\check{\mathbb{P}}^{2}$. Since the variables $v_{0}, v_{1}, v_{2}$ are associated to the filtrations $W, F^{\prime}, F^{\prime \prime}$ in (1.3.1), we denote the coordinate lines in $\breve{\mathbb{P}}^{2}$ by

$$
\check{\mathbb{P}}_{W}^{1}=\left\{v_{0}=0\right\}, \quad \check{\mathbb{P}}_{F^{\prime}}^{1}=\left\{v_{1}=0\right\}, \quad \check{\mathbb{P}}_{F^{\prime \prime}}^{1}=\left\{v_{2}=0\right\},
$$

and the torus fixed points by

$$
\check{\mathbb{P}}_{W F^{\prime}}^{0}=\left\{v_{0}=v_{1}=0\right\}, \quad \check{\mathbb{P}}_{W F^{\prime \prime}}^{0}=\left\{v_{0}=v_{2}=0\right\}, \quad \check{\mathbb{P}}_{F^{\prime} F^{\prime \prime}}^{1}=\left\{v_{1}=v_{2}=0\right\} .
$$

Then the restrictions of the Rees bundles to the coordinate lines are found as follows (see [14], 2.6.2):

$$
\left.\mathcal{E}\right|_{\check{\mathbb{P}}_{W}^{1}}=\bigoplus_{n} \mathbb{P R s}\left(\operatorname{gr}_{n}^{W} V,{F^{\prime}}^{\bullet}, F^{\prime \prime \bullet}\right) \otimes \mathcal{O}(-n)
$$

Here on the right-hand side we have the Rees bundles on $\mathbb{P}^{1}$ corresponding to the bifiltered spaces $\left(\mathrm{gr}_{n}^{W} V,{F^{\prime}}^{\circ}, F^{\prime \prime \bullet}\right.$ ) (induced filtrations). Similarly for other coordinate lines. The restrictions to (i.e., fibers over) the fixed points are given by the bigraded spaces associated to the corresponding pairs of filtrations:

$$
\left.\mathcal{E}\right|_{\widetilde{\mathbb{P}}_{W F^{\prime}}^{0}}=\mathrm{gr}_{\bullet}^{W} \operatorname{gr}_{F^{\prime}}^{\cdot}(V),
$$

etc. Now recall an observation of Deligne, used by Simpson [16].

Proposition 1.3.4. Let $F^{\bullet}, F^{\prime \prime}$ be two filtrations on a finite-dimensional vector space $V$. Then the following are equivalent:

(i) $F^{\circ}$ and $F^{\prime \prime \bullet}$ are $n$-opposite (induce a pure complex Hodge structure of weight $n$ ).

(ii) The Rees bundle $\mathbb{P R s}\left(V, F^{\prime \bullet}, F^{\prime \prime}\right)$ on $P^{1}$ is a direct sum of several copies of $\mathcal{O}(n)$.

As observed in [14], the isomorphism (1.3.2) implies that for a complex mixed Hodge structure $\left(V, W_{\bullet},{F^{\prime}}^{\bullet}, F^{\prime \prime \bullet}\right)$ the restriction $\left.\mathcal{E}\right|_{\check{\mathbb{P}}_{W}^{1}}$ is trivial:

$$
\left.\mathcal{E}\right|_{\widetilde{\mathbb{P}}_{W}^{1}} \simeq \operatorname{gr}_{\cdot}^{W}(V) \otimes \mathcal{O}_{\check{\mathbb{P}}_{W}^{1}}
$$


Denote by $\mathcal{P}$ the following category. Objects of $\mathcal{P}$ are $\mathbb{G}_{m}^{2}$-equivariant vector bundles $\mathcal{E}$ on $\mathbb{P}^{2}$ which are trivial on $\widetilde{\mathbb{P}}_{W}^{1}$. Morphisms in $\mathcal{P}$ are equivariant morphisms of bundles which have constant rank everywhere except possibly the point $\breve{\mathbb{P}}_{F^{\prime} F^{\prime \prime}}^{0}$. Clearly, $\mathcal{P}$ is a tensor category with respect to the tensor product of vector bundles. The main result of [13], [14] is (see [13], Theorem 3.1):

Theorem 1.3.6. The Rees bundle construction defines an equivalence of tensor categories

$$
\mathrm{MHS}_{\mathbb{C}} \rightarrow \mathcal{P}
$$

Now notice the following:

Lemma 1.3.7. The categories $\mathcal{P}$ and $\mathcal{Q}$ are equivalent.

Proof. We first identify the objects. On the surface of it, $\mathcal{P}$ seems to have more objects, as we require triviality on one line only rather than on all lines not meeting $\check{\mathbb{P}}_{F^{\prime} F^{\prime \prime}}^{0}$. However, triviality is an open condition for vector bundles on $\mathbb{P}^{1}$. So if $\mathcal{E} \in \mathcal{P}$ is trivial on $\breve{\mathbb{P}}_{W}^{1}$, it is trivial on an open subset $U$ of lines in $\breve{\mathbb{P}}^{2}$ containing $\check{\mathbb{P}}_{W}^{1}$. By equivariance, $U$ can be assumed to be preserved under the torus action. This implies that $U$ contains all lines not meeting $\check{\mathbb{P}}_{F^{\prime} F^{\prime \prime}}^{0}$. So $\mathcal{E}$ is an object of $\mathcal{Q}$ as well.

Next, we identify the morphisms. On the surface of it, $Q$ seems to have more morphisms, as we do not require the constant rank condition. So let $f: \mathcal{E} \rightarrow \mathcal{E}^{\prime}$ be a morphism in $\mathcal{Q}$, i.e., just an invariant morphism of equivariant vector bundles, both being objects of $\mathcal{Q}$. Let $\lambda \subset \mathbb{P}^{2}$ be a projective line such that both $\mathcal{E}$ and $\mathcal{E}^{\prime}$ are trivial on $\lambda$. Then clearly $f$ has constant rank along $\lambda$. On the other hand, any two points of $\breve{\mathbb{P}}^{2}-\breve{\mathbb{P}}_{F^{\prime} F^{\prime \prime}}^{0}$ are connected by a chain of lines $\lambda$ as above. This implies that the rank of $f$ is constant on $\breve{\mathbb{P}}^{2}-\breve{\mathbb{P}}_{F^{\prime} F^{\prime \prime}}^{0}$, so $f$ is a morphism of $\mathcal{P}$.

1.4. Proof of Theorems 1.1.4 and 0.3 (a)-(d). Combining now Theorems 1.2.7, 1.3.6 and Lemma 1.3.7, we get an equivalence of tensor categories

$$
\mathrm{MHS}_{\mathbb{C}} \stackrel{\mathbb{P R s}}{\longrightarrow} \mathcal{Q}=\mathcal{P} \stackrel{\Psi}{\rightarrow} \operatorname{Bun}_{\nabla}\left(\mathbb{A}^{2} ; \mathbb{G}_{m}^{2}\right),
$$

where $\mathbb{P R s}$ is the Rees bundle construction, and $\Psi$ is the inverse Radon-Penrose transform. This is the equivalence claimed in Theorem 1.1.4. To finish the proof, it is enough to compare the behavior of the equivalence with respect to the $\Gamma$-action, see Section 1.1. The definition (1.1.2) of the conjugate Hodge structure implies that the coordinates $v_{0}, v_{1}, v_{2}$ on $\breve{\mathbb{P}}^{2}$ associated to the three filtrations in the Rees construction, are transformed under $\sigma$ as follows:

$$
v_{0} \mapsto \bar{v}_{0}, \quad v_{1} \mapsto \bar{v}_{2}, \quad v_{2} \mapsto \bar{v}_{1} .
$$

This translates into the action (1.1.3) on the plane $\mathbb{A}^{2}$ formed by lines in $\check{\mathbb{P}}^{2}$ not meeting $\check{\mathbb{P}}_{F^{\prime} F^{\prime \prime}}^{0}$. This finishes the proof of Theorem 1.1.4 and thus of Theorem 0.3 (a). 
To see part (b) of Theorem 0.3, note that by general properties of the inverse Radon-Penrose transform, see (1.2.8), and by (1.3.5), we have

$$
E_{0}=H^{0}\left(\check{\mathbb{P}}_{W}^{1}, \mathcal{E}\right)=\operatorname{gr}_{\bullet}^{W}(V) .
$$

Next, to see part (c), note that by the definitions of the Rees module and bundle, $V$ is recovered as the fiber

$$
V=\operatorname{Rs}\left(V, W_{\bullet}, F^{\prime \bullet}, F^{\prime \prime \bullet}\right) /\left(v_{1}-1, v_{2}-1, v_{3}-1\right)=\mathcal{E}_{[1: 1: 1]} .
$$

The point $[1: 1: 1] \in \breve{\mathbb{P}}^{2}$ corresponds to the line

$$
\left\{1+t_{1}+t_{2}=0\right\} \subset \mathbb{A}^{2},
$$

which, in the presence of the real structure $t_{1}=t, t_{2}=\bar{t}$, can be described as $\operatorname{Re}(t)=-1 / 2$. Our statement then follows from the definition of the Radon-Penrose transform, see Proposition 1.2.3.

To see part (d), notice that flatness of $\nabla$ is equivalent to the property that $\varepsilon^{0}$ (and thus $\mathcal{E}$ ) is trivial as a vector bundle. The fact that triviality of the Rees bundle is equivalent to splitting of the Hodge structure, was pointed out in [14], (2.10), Theorem 2.

\subsection{Noncommutative differential operators and absolute Hodge cohomology.} Here we prove part (d) of Theorem 0.3. We will prove the following complex version. Taking into account the real structures is straightforward.

Theorem 1.5.1. If $(E, \nabla)$ is the $\mathbb{G}_{m}^{2}$-equivariant bundle with connection on $\mathbb{A}^{2}$ corresponding to a complex Hodge structure $\left(V, W_{\bullet}, F^{\prime \bullet}, F^{\prime \prime}\right)$, then we have a natural quasiisomorphism

$$
\Gamma\left(\mathbb{A}^{2},\left\{E \stackrel{\nabla}{\rightarrow} E \otimes \Omega_{\mathbb{A}^{2}}^{1}\right\}\right)^{\mathbb{G}_{m}^{2}} \sim R \operatorname{Hom}_{\mathrm{MHS}_{\mathbb{C}}}(\mathbb{C}(0), V) .
$$

For the proof we embed $\operatorname{Bun}_{\nabla}\left(\mathbb{A}^{2} ; \mathbb{G}_{m}^{2}\right)$ into a larger abelian category in which the trivial bundle $\mathcal{O}_{\mathbb{A}^{2}}$ (which corresponds to the Hodge structure $\mathbb{C}(0)$ ) has a projective resolution.

Recall [10] that for any smooth algebraic variety $X / \mathbb{C}$ there is a sheaf $\mathbb{D}_{X}$ of noncommutative rings on $X$ called the sheaf of noncommutative differential operators. We will need the following properties of $\mathbb{D}_{X}$. First, $\mathbb{D}_{X}$ has a multiplicative filtration $\left\{\mathbb{D}_{\bar{X}}^{\leq d}\right\}$ (by “order") with quotients identified with

$$
\mathbb{D}_{\bar{X}}^{\leq d} / \mathbb{D}_{\bar{X}}^{\leq d-1} \simeq T_{X}^{\otimes d},
$$

so that the associated graded algebra of $\mathbb{D}_{X}$ is the tensor algebra of $T_{X}$.

Second, there is a natural embedding

$$
\epsilon: T_{X} \rightarrow \mathbb{D}_{\bar{X}}^{\leq 1},
$$


splitting the first level of the filtration.

Third, $\mathbb{D}_{X}$ plays the same role for nonflat connections as the ordinary sheaf of differential operators does for flat ones. To be precise, we have the following.

Proposition 1.5.2. Let $E$ be any quasi-coherent sheaf of $\mathcal{O}_{X}$-modules. Then structures of a $\mathbb{D}_{X}$-module on $E$ extending the $\mathcal{O}_{X}$-module structure are in bijection with connections (flat or not) on $E$.

Example 1.5.3. Let $X=\mathbb{A}^{n}$ with coordinates $t_{1}, \ldots, t_{n}$. Then the ring $\mathbb{D}\left(\mathbb{A}^{n}\right)$ is generated by the polynomial ring $\mathbb{C}\left[t_{1}, \ldots, t_{n}\right]$ and by the symbols $\nabla_{1}, \ldots, \nabla_{n}$, which are required to satisfy

$$
\left[\nabla_{i}, t_{j}\right]=\delta_{i j}, \quad 1 \leq i, j \leq n,
$$

and no other relations. In particular, $\nabla_{1}, \ldots, \nabla_{n}$ generate a free associative algebra. Given a bundle with connection $(E, \nabla)$ on $\mathbb{A}^{n}$, the generator $\nabla_{i}$ acts in $E$ by the covariant derivative $\nabla_{\partial / \partial t_{i}}$.

Proposition 1.5.4. Let $E^{\prime}$ be any sheaf of $\mathbb{D}_{X}$-modules quasi-coherent over $\mathcal{O}_{X}$ (i.e., a quasi-coherent $\mathcal{O}_{X}$-module with a connection). Then the following 2-term version of the Spencer sequence is a locally free left $\mathbb{D}_{X}$-resolution of $E^{\prime}$ :

$$
\begin{aligned}
\mathcal{S}^{\bullet}\left(E^{\prime}\right) & =\left\{\mathbb{D}_{X} \otimes \mathcal{O}_{X} T_{X} \otimes \mathcal{O}_{X} E^{\prime} \stackrel{d}{\rightarrow} \mathbb{D}_{X} \otimes \mathcal{O}_{X} E^{\prime}\right\}, \\
d\left(P \otimes v \otimes e^{\prime}\right) & =P \cdot \epsilon(v) \otimes e^{\prime}-P \otimes \epsilon(v)\left(e^{\prime}\right) .
\end{aligned}
$$

Proof. Filtering $\varsigma^{\bullet}\left(E^{\prime}\right)$ by the subcomplexes

$$
\left\{\mathbb{D}_{\bar{X}}^{\leq(d-1)} \otimes_{\mathcal{O}_{X}} T_{X} \otimes \mathcal{O}_{X} E^{\prime} \stackrel{d}{\rightarrow} \mathbb{D}_{\bar{X}}^{\leq d} \otimes_{\mathcal{O}_{X}} E^{\prime}\right\}, \quad d \geq 0,
$$

we find the associated graded complex to be

$$
\left(\bigoplus_{d \geq 0} T_{X}^{\otimes(d-1)}\right) \otimes T_{X} \otimes E^{\prime} \rightarrow \bigoplus_{d \geq 0} T_{X}^{\otimes d} \otimes E^{\prime} .
$$

Since the associated graded algebra of $\mathbb{D}_{X}$ is the tensor algebra of $T_{X}$, we see that the differential in (1.5.5) is the tensor multiplication, and so it is a resolution of $T_{X}^{\otimes 0} \otimes E^{\prime}=E^{\prime}$ as a left module over the tensor algebra. Using the exactness of the associated graded complex (1.5.5), we deduce the exactness of $\delta^{\bullet}\left(E^{\prime}\right)$ by a spectral sequence argument.

Let now $\mathcal{M}$ be the category of $\mathbb{G}_{m}^{2}$-equivariant quasi-coherent sheaves of $\mathcal{O}_{\mathbb{A}^{2-}}$ modules, equipped with an equivariant connection (not necessarily flat). In other

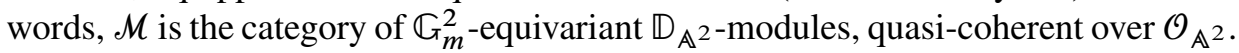
Clearly, $\mathcal{M}$ is an abelian category. Theorem 1.1.4 realizes $\mathrm{MHS}_{\mathbb{C}}$ as a full subcategory in $\mathcal{M}$ (formed by quasi-coherent sheaves which are vector bundles). Note that this subcategory is also abelian, and is closed under extensions in $\mathcal{M}$. This implies that 
for any two objects $V, V^{\prime} \in \mathrm{MHS}_{\mathbb{C}}$ with the corresponding objects $E, E^{\prime} \in \mathcal{M}$, the natural morphism

$$
\operatorname{Ext}_{\mathrm{MHS}_{\mathbb{C}}^{i}}\left(V^{\prime}, V\right) \rightarrow \operatorname{Ext}_{\mathcal{M}}^{i}\left(E^{\prime}, E\right)
$$

is an isomorphism for $i=0,1$.

Proposition 1.5.6. (a) The category $\mathrm{MHS}_{\mathbb{C}}$ has cohomological dimension 1, i.e., for any two objects $V, V^{\prime}$ we have $\operatorname{Ext}_{\mathrm{MHS}}^{i}\left(V^{\prime}, V\right)=0$ for $i \geq 2$.

(b) The category $\mathcal{M}$ also has cohomological dimension 1 . Therefore for any two objects $V^{\prime}, V \in \mathrm{MHS}_{\mathbb{C}}$ with corresponding equivariant bundles with connections $E^{\prime}, E$ the natural morphism

$$
R \operatorname{Hom}_{\mathrm{MHS}}\left(V^{\prime}, V\right) \rightarrow R \operatorname{Hom}_{\mathcal{M}}\left(E^{\prime}, E\right)
$$

is a quasi-isomorphism.

Proof. Part (a) was proved by Carlson [2]. Let us prove part (b). Given two objects $E, E^{\prime}$ of $\mathcal{M}$, the complex of vector spaces $R \operatorname{Hom}_{\mathcal{M}}\left(E^{\prime}, E\right)$ is obtained from the complex

$$
\underline{R \operatorname{Hom}}_{\mathbb{D}_{\mathbb{A}^{2}}}\left(E^{\prime}, E\right)=\underline{\operatorname{Hom}}_{\mathbb{D}_{\mathbb{A}^{2}}}\left(\varsigma^{\bullet}\left(E^{\prime}\right), E\right)
$$

of sheaves on $\mathbb{A}^{2}$ by taking (the derived functor of) global sections and then taking the (derived functor of the) subspace of invariants with respect to $\mathbb{G}_{m}^{2}$. Now, the complex (1.5.7) is a 2-term complex of quasi-coherent sheaves on $\mathbb{A}^{2}$, equivariant with respect to $\mathbb{G}_{m}^{2}$. Since $\mathbb{A}^{2}$ is affine and $\mathbb{G}_{m}^{2}$ is reductive, we do not need to derive the functors of global sections and invariants, so $R \operatorname{Hom}\left(E^{\prime}, E\right)$ is still a 2-term complex.

This implies Theorem 1.5.1. Indeed, the statement and the proof of Proposition 1.5.6 imply that

$$
R \operatorname{Hom}_{\mathrm{MHS}_{\mathbb{C}}}(\mathbb{C}(0), V)=R \operatorname{Hom}_{\mathcal{M}}\left(\mathcal{O}_{\mathbb{A}^{2}}, E\right)=\Gamma\left(\mathbb{A}^{2}, \underline{\operatorname{Hom}}_{\mathbb{D}_{\mathbb{A}^{2}}}\left(S^{\bullet}\left(\mathcal{O}_{\mathbb{A}^{2}}\right), E\right)\right)^{\mathbb{G}_{m}^{2}},
$$

which is nothing but the global equivariant de Rham sequence in the formulation of the theorem.

\section{The Deligne operator as the holonomy of an equivariant connection}

2.1. Reminder on the Deligne operator. We start by recalling the description of $\mathrm{MHS}_{\mathbb{C}}$ by means of the "Hodge monodromy" operator $\delta$ given by Deligne [7]. For any object $\left(V, W_{\bullet}, F^{\prime \bullet}, F^{\prime \prime}\right)$, there are two splittings

$$
V=\bigoplus_{p, q \in \mathbb{Z}} V_{F^{\prime}}^{p, q}=\bigoplus_{p, q \in \mathbb{Z}} V_{F^{\prime \prime}}^{p, q},
$$


(see [7], p. 510) with the following properties. First, both these splittings induce the same filtration $W$. by

$$
\bigoplus_{p+q \leq n} V_{F^{\prime}}^{p, q}=\bigoplus_{p+q \leq n} V_{F^{\prime \prime}}^{p, q}=W_{n}(V) .
$$

Secondly, the first splitting induces ${F^{\prime}}^{\bullet}$ and the second $F^{\prime \prime}$ by

$$
F^{\prime p}(V)=\bigoplus_{p^{\prime} \geq p, q \in \mathbb{Z}} V_{F^{\prime}}^{p, q}, \quad F^{\prime \prime q}(V)=\bigoplus_{p \in \mathbb{Z}, q^{\prime} \geq q} V_{F^{\prime \prime}}^{p, q^{\prime}} .
$$

Thirdly,

$$
V_{F^{\prime}}^{q, p} \equiv \sigma^{-1}\left(\sigma(V)_{F^{\prime \prime}}^{p, q}\right) \bmod W_{p+q-1}(V) .
$$

Here $\sigma$ is the antilinear isomorphism (1.1.1) and $\sigma(V)_{F^{\prime \prime}}^{p, q}$ is the second splitting but for the conjugate Hodge structure as defined by (1.1.2). Let us also denote for simplicity

$$
\operatorname{gr}^{p, q}(V)=\operatorname{gr}_{F^{\prime}}^{p} \operatorname{gr}_{F^{\prime \prime}}^{q} \operatorname{gr}_{p+q}^{W}(V)
$$

Then the projections

$$
a_{F^{\prime}}^{p, q}: V_{F^{\prime}}^{p, q} \rightarrow \mathrm{gr}^{p, q}(V), \quad a_{F^{\prime \prime}}^{p, q}: V_{F^{\prime \prime}}^{p, q} \rightarrow \mathrm{gr}^{p, q}(V)
$$

are isomorphisms. So their direct sums, denoted by $a_{F^{\prime}}$ and $a_{F^{\prime \prime}}$, are isomorphisms

$$
a_{F^{\prime}}, a_{F^{\prime \prime}}: V \rightarrow \operatorname{gr}_{\bullet}^{W}(V)=\bigoplus_{p, q} \mathrm{gr}^{p, q}(W),
$$

and so we have the automorphism

$$
\delta=a_{F^{\prime \prime}} a_{F^{\prime}}^{-1}: \operatorname{gr}_{\bullet}^{W}(V) \rightarrow \operatorname{gr}_{\bullet}^{W}(V),
$$

which is known to satisfy

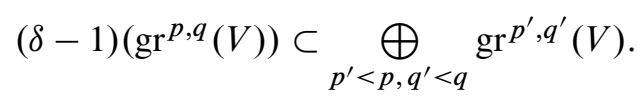

Deligne's characterization is then as follows.

Theorem 2.1.5. Let $\Delta$ be the category of pairs $\left(V^{\bullet \bullet}, \delta\right)$, where $V^{\bullet \bullet}=\bigoplus V^{p, q}$ is a finite-dimensional bigraded $\mathbb{C}$-vector space and $\delta$ is an automorphism of $V^{\bullet \bullet}$ satisfying

$$
(\delta-1)\left(V^{p, q}\right) \subset \underset{p^{\prime}<p, q^{\prime}<q}{\bigoplus^{p^{\prime}, q^{\prime}}}
$$

Then the functor

$$
\left(V, W_{\bullet}, F^{\prime \bullet}, F^{\prime \prime \bullet}\right) \mapsto\left(V^{\bullet \bullet}=\mathrm{gr}^{\bullet \bullet}(V), \delta=a_{F^{\prime \prime}} a_{F^{\prime}}^{-1}\right)
$$

is an equivalence of tensor categories $\mathrm{MHS}_{\mathbb{C}} \rightarrow \Delta$. 
2.2. The Hodge group. Now, still following [7], we reformulate Theorem 2.1 .5 in terms of representations of appropriate Lie algebras and groups. Indeed, $\delta$ being unipotent, specifying $\delta$ is equivalent to specifying its logarithm

$$
D=\log (\delta)=\sum_{p, q \geq 1} D_{p, q},
$$

where $D_{p, q}$ is the bihomogeneous part of degree $(-p,-q)$, i.e., $D_{p, q}\left(V^{p^{\prime}, q^{\prime}}\right) \subset$ $V^{p^{\prime}-p, q^{\prime}-q}$ for any $p^{\prime}, q^{\prime}$. Now, the operators $D_{p, q}$ can be given arbitrarily subject only to the homogeneity conditions. So we get:

Reformulation 2.2.1. $\mathrm{MHS}_{\mathbb{C}}$ is equivalent to the category of finite-dimensional bigraded representations of the free Lie algebra

$$
\mathfrak{L}=\operatorname{FLie}\left\{z_{p, q} \mid p, q \geq 1\right\},
$$

where the generator $z_{p, q}$ has bidegree $(-p,-q)$.

The bigrading in $\mathcal{Q}$ induces the weight filtration $W$ on it, as in (2.1.2), and this filtration is compatible with the Lie algebra structure. Each quotient $\mathfrak{L} / W_{-d} \mathfrak{R}$ is finitedimensional and nilpotent. Denote by $\exp \left(\mathfrak{L} / W_{-d} \mathfrak{L}\right)$ the corresponding unipotent algebraic group. We have then the pro-algebraic group

$$
\mathfrak{U}=\lim _{d} \exp \left(\mathfrak{L} / W_{-d} \mathfrak{R}\right)
$$

The bigrading on $\mathfrak{L} / W_{-d} \mathfrak{R}$ can be interpreted as an action of $\mathbb{G}_{m}^{2}$, and these actions induce an action on $\mathfrak{U}$, so we have the semidirect product

$$
\mathfrak{s}=\mathbb{G}_{m}^{2} \times \mathcal{U} .
$$

Then, MHS $\mathbb{C}$ is equivalent to the category of finite-dimensional representations of (5). The group $\mathbb{G}$, sometimes referred to as the Hodge group, is the group of automorphisms of the fiber functor

$$
\omega_{W}: \operatorname{MHS}_{\mathbb{C}} \rightarrow \operatorname{Vect}_{\mathbb{C}}, \quad\left(V, W_{\bullet}, F^{\prime \bullet}, F^{\prime{ }^{\bullet}}\right) \mapsto \operatorname{gr}^{\bullet \bullet}(V) .
$$

2.3. Comparison of two descriptions. We now compare the description of $\mathrm{MHS}_{\mathbb{C}}$ given by Theorem 2.1.5 (or, equivalently, by Reformulation 2.2.1) with the description of Theorem 1.1.4 via equivariant connections.

Theorem 2.3.1. Let $\left(V, W_{\bullet},{F^{\prime}}^{\bullet}, F^{\prime \prime \bullet}\right) \in \operatorname{MHS}_{\mathbb{C}}$, and $(E, \nabla)$ be the corresponding $\mathbb{G}_{m}^{2}$-equivariant bundle with connection in $\mathbb{A}^{2}$. Then, under the identification $E_{0}=$ $\mathrm{gr}^{* \bullet}(V)$ given by (1.4.1), the Deligne operator

$$
\delta: E_{(0,0)}=V^{\bullet \bullet} \rightarrow V^{\bullet \bullet}=E_{(0,0)}
$$

is recovered as the holonomy of $\nabla$ along the boundary of the triangle with vertices $(0,0),(-1,0),(0,-1)$. 
Proof. We realize the bundle $\mathcal{E}$ explicitly by a patching function. We represent the punctured projective plane as the union of two affine planes:

$$
\check{\mathbb{P}}_{0}^{2}=\check{\mathbb{P}}^{2}-\check{\mathbb{P}}_{F^{\prime} F^{\prime \prime}}^{0}=\check{\mathbb{A}}_{F^{\prime}}^{2} \cup \check{\mathbb{A}}_{F^{\prime \prime}}^{2},
$$

where

$$
\begin{array}{lll}
\check{\mathbb{A}}_{F^{\prime}}^{2}=\operatorname{Spec} \mathbb{C}\left[\xi_{0}, \xi_{1}\right], & \xi_{0}=v_{0} / v_{2}, & \xi_{1}=v_{1} / v_{2}, \\
\check{\mathbb{A}}_{F^{\prime \prime}}^{2}=\operatorname{Spec} \mathbb{C}\left[\eta_{0}, \eta_{2}\right], & \eta_{0}=v_{0} / v_{1}, & \eta_{2}=v_{2} / v_{1},
\end{array}
$$

are the affine charts centered at the points $\check{\mathbb{P}}_{W F^{\prime}}^{0}$ and $\check{\mathbb{P}}_{W F^{\prime \prime}}^{0}$. We are interested only in the restriction $\mathcal{E}^{0}$ of $\&$ to $\breve{\mathbb{P}}_{0}^{2}$, as points of $\mathbb{A}^{2}$ correspond to lines contained in $\breve{\mathbb{P}}_{0}^{2}$.

The restriction of the Rees bundle $\mathcal{E}$ to $\check{\mathbb{A}}_{F}^{2}$, is just the Rees bundle on $\mathbb{A}^{2}$ corresponding to the pair of filtrations $W, F^{\prime}$; see [14], 2.6.1. In other words, this is the bundle corresponding to the $\mathbb{C}\left[\xi_{0}, \xi_{1}\right]$-module

$$
M_{F^{\prime}}=\bigoplus_{i, j}\left(W_{i} \cap F^{\prime-j}\right)(V) \xi_{0}^{i} \xi_{1}^{j} \subset V\left[\xi_{0}^{ \pm 1}, \xi_{1}^{ \pm 1}\right] .
$$

Note that $W_{\bullet}, F^{\bullet}$ are simultaneously split by the first bigrading $\left(V_{F^{\prime}}^{p, q}\right)$ from (2.1.1), so by (2.1.3) we can identify $M_{F^{\prime}}$ with a free module as follows:

$$
M_{F^{\prime}}=\bigoplus_{i, j} \bigoplus_{\substack{p+q \leq i \\ p \geq-j}} V_{F^{\prime}}^{p, q} \xi_{0}^{i} \xi_{1}^{j}=\bigoplus_{p, q} V_{F^{\prime}}^{p, q} \otimes \xi_{0}^{p+q} \xi_{1}^{-p} \mathbb{C}\left[\xi_{0}, \xi_{1}\right]
$$

In other words, we have the trivialization

$$
\begin{aligned}
& \tau_{F^{\prime}}: \operatorname{gr}^{\bullet \bullet}(V) \otimes \mathbb{C}\left[\xi_{0}, \xi_{1}\right] \rightarrow M_{F^{\prime}}, \\
& \tau_{F^{\prime}}\left(v_{p q} \otimes f\left(\xi_{0}, \xi_{1}\right)\right)=a_{F^{\prime}}^{-1}\left(v_{p q}\right) \otimes \xi_{0}^{p+q_{1}} \xi_{1}^{-p} f\left(\xi_{0}, \xi_{1}\right), v_{p, q} \in \operatorname{gr}^{p, q}(V) .
\end{aligned}
$$

Here $a_{F^{\prime}}^{-1}$ is the inverse of the isomorphism induced by the splitting $\left(V_{F}^{p, q}\right)$, see (2.1.4).

Similarly, the restriction of $\mathcal{E}$ to $\breve{\mathbb{A}}_{F}^{2}$, is the Rees bundle on $\mathbb{A}^{2}$ corresponding to $W, F^{\prime \prime}$, so it corresponds to the $\mathbb{C}\left[\eta_{0}, \eta_{2}\right]$-module

$$
M_{F^{\prime \prime}}=\bigoplus_{k, l}\left(W_{k} \cap F^{\prime \prime-l}\right)(V) \eta_{0}^{k} \eta_{2}^{l}=\bigoplus_{k, l} \underset{\substack{p+q \leq k \\ q \geq-l}}{\bigoplus} V_{\bar{F}}^{p, q} \eta_{0}^{k} \eta_{2}^{l}
$$

where we now used the second splitting in (2.1.1). So we get a trivialization

$$
\begin{aligned}
& \tau_{F^{\prime \prime}}: \mathrm{gr} \bullet(V) \otimes \mathbb{C}\left[\eta_{0}, \eta_{2}\right] \rightarrow M_{F^{\prime \prime}}, \\
& \tau_{F^{\prime \prime}}\left(v_{p q} \otimes g\left(\eta_{0}, \eta_{2}\right)\right)=a_{F^{\prime \prime}}^{-1}\left(v_{p, q}\right) \otimes \eta_{0}^{p+q} \eta_{2}^{-q} g\left(\eta_{0}, \eta_{2}\right) .
\end{aligned}
$$

This means that we have glued $\mathcal{E}^{0}$ out of two trivial bundles, each with fiber gr ${ }^{\bullet \bullet}(V)$. Let us view a point $\xi=\left(\xi_{0}, \xi_{1}\right) \in \breve{\mathbb{A}}_{F}^{2}$, with both coordinates nonzero as an element 
of the torus $\mathbb{G}_{m}^{2}$. Let $\xi \mapsto \phi(\xi)$ be the torus action on gr“ $(V)$ corresponding to the bigrading. In coordinates $\xi_{0}, \xi_{1}$ it has the form

$$
\phi(\xi)\left(v_{p, q}\right)=\xi_{0}^{p+q} \xi_{1}^{-p} v_{p, q}, \quad v_{p, q} \in \mathrm{gr}^{p, q}(V) .
$$

Writing now the identification of these two bundles on $\check{\mathbb{A}}_{F^{\prime}}^{2} \cap \check{\mathbb{A}}_{F^{\prime \prime}}^{2}$, i.e., expressing the $\eta$ 's through the $\xi$ 's and accounting for the monomial factors in (2.3.2) and (2.3.3), we find straightforwardly:

Proposition 2.3.4. The patching function $\Phi=\tau_{\bar{F}}^{-1} \tau_{F}$ of $\mathcal{E}^{0}$ with respect to the above trivializations has the form

$$
\Phi(\xi)=\phi(\xi)^{-1} \delta \phi(\xi)
$$

We now prove Theorem 2.3.1. Recall that each point $T=\left(t_{1}, t_{2}\right) \in \mathbb{A}^{2}$ corresponds to a line

$$
\lambda_{T}=\left\{v_{0}+t_{1} v_{1}+t_{2} v_{1}=0\right\} \subset \check{\mathbb{P}}_{0}^{2} .
$$

Given two distinct points $T, T^{\prime}$, we denote by $\left[T, T^{\prime}\right]$ the straight line segment joining them. Since $\mathcal{E}$ is trivial on each $\lambda_{T}$, the restriction map

$$
R_{T T^{\prime}}: E_{T}=H^{0}\left(\lambda_{T}, \mathscr{E}\right) \rightarrow \mathcal{E}_{\lambda_{T} \cap \lambda_{T^{\prime}}}=H_{\nabla}^{0}\left(\left[T, T^{\prime}\right], E\right)
$$

is an isomorphism. By general properties of the Radon-Penrose transform (cf., e.g., [17], p. 377), the holonomy along $\left[T, T^{\prime}\right]$ is found as

$$
H_{T T^{\prime}}=R_{T^{\prime} T}^{-1} \circ R_{T T^{\prime}}: E_{T} \rightarrow E_{T^{\prime}} .
$$

Now notice that $\lambda_{(0,0)}=\check{\mathbb{P}}_{W}^{1}$, while $\lambda_{(-1,0)}$ is the line $\left\{v_{0}=v_{1}\right\}$ joining the points $\breve{\mathbb{P}}_{W F}^{0}=[0: 0: 1]$ and $[1: 1: 1]$. Similarly, $\lambda_{(0,-1)}$ is the line $\left\{v_{0}=v_{2}\right\}$ joining $\breve{\mathbb{P}}_{W \bar{F}}^{0}=[0: 0: 1]$ and $[1: 1: 1]$. By Proposition 2.3 .4 , the value of the patching function $\Phi(\xi)$ at the point $\xi_{0}=\xi_{1}=1$, which is the same as [1:1:1], is equal to $\delta$. This implies:

Lemma 2.3.5. If we identify $H^{0}\left(\lambda_{(-1,0)}, \mathcal{E}\right)$ and $H^{0}\left(\mathcal{E}, \lambda_{(0,-1)}, \mathcal{E}\right)$ with gr"• $(V)$, using the trivializations $\tau_{F^{\prime}}$ and $\tau_{F^{\prime \prime}}$, then the composite isomorphism

$$
H^{0}\left(\lambda_{(-1,0)}, \mathcal{E}\right) \stackrel{R_{(-1,0),(0,-1)}}{\longrightarrow} \mathcal{E}_{[1: 1: 1]} \stackrel{R_{(0,-1),(-1,0)}^{-1}}{\longrightarrow} H^{0}\left(\lambda_{(0,-1)}, \mathcal{E}\right)
$$

is identified with $\delta$.

The lemma implies Theorem 2.3.1 since with respect to our identifications the holonomies along the two other sides of the triangle are equal to 1 . 
2.4. Equivariant connections in coordinates. Let $V^{\bullet \bullet}$ be a finite-dimensional bigraded $\mathbb{C}$-vector space, and let $\widetilde{V}^{\bullet \bullet}$ be the corresponding $\mathbb{G}_{m}^{2}$ - equivariant vector bundle on $\mathbb{A}^{2}$. As a vector bundle, it is trivial: $\widetilde{V}^{\bullet \bullet}=V^{\bullet \bullet} \otimes \mathcal{O}_{\mathbb{A}^{2}}$, while the $\mathbb{G}_{m}^{2}$ action is given by

$$
\left(\lambda_{1}, \lambda_{2}\right)\left(v_{p q} \otimes f\left(t_{1}, t_{2}\right)\right)=t_{1}^{p} t_{2}^{q} v_{p q} \otimes f\left(\lambda_{1} t_{1}, \lambda_{2} t_{2}\right), \quad v_{p q} \in V^{p, q} .
$$

Lemma 2.4.1. Every $\mathbb{G}_{m}^{2}$-equivariant vector bundle on $\mathbb{A}^{2}$ is equivariantly isomorphic to a bundle of the form $\widetilde{V}^{* \bullet}$.

Proof. It is well known that every $\mathbb{G}_{m}^{2}$-equivariant vector bundle on $\mathbb{A}^{2}$ is the Rees bundle corresponding to two filtrations; see, e.g., [14], (2.5.3), Proposition 14. Our statement follows from the fact that any two filtrations can be simultaneously split by a bigrading, see, e.g., [14], (2.1.3), Lemme 1.

Proposition 2.4.2. Any $\mathbb{G}_{m}^{2}$-invariant connection in $\tilde{V}^{\bullet \bullet}$ has the form

$$
\nabla=d+\Omega, \quad \Omega=\sum_{p, q \geq 1} A_{p, q} t_{1}^{p-1} t_{2}^{q} d t_{1}+B_{p, q} t_{1}^{p} t_{2}^{q-1} d t_{2},
$$

where $d$ is the standard flat connection of the trivial bundle $\tilde{V}^{\bullet \cdot}$ and $A_{p, q}, B_{p, q}$ are endomorphisms of $V^{* \bullet}$ of bidegree $(-p,-q)$.

Proof. The space of $d$-covariantly constant (i.e., constant) sections of $\tilde{V}^{* \bullet}$ is preserved by the action $\mathbb{G}_{m}^{2}$ (although individual elements of this space may not be). Next, the datum of any connection at a given point $x \in \mathbb{A}^{2}$ is given by the subspace $C_{x}$ in the space of germs of sections near $x$ which are covariantly constant up to the first order of tangency. Looking at the trivial connection $d$, we see, just as for global sections, that the action of $\lambda \in \mathbb{G}_{m}^{2}$ takes the subspace $C_{x}$ into the subspace $C_{\lambda(x)}$. This means that $d$ is in fact a $\mathbb{G}_{m}^{2}$-invariant connection in $\tilde{V}^{\bullet \bullet}$. Therefore any other invariant connection has the form $d+\Omega$, where $\Omega$ is a global 1 -form on $\mathbb{A}^{2}$ with values in $\operatorname{End}\left(\widetilde{V}^{\bullet \bullet}\right)$, which is $\mathbb{G}_{m}^{2}$-invariant, i.e., has total degree 0 . The sum in the proposition is nothing but the general shape of such a 1 -form.

We denote by

$$
W_{n}\left(V^{\bullet \bullet}\right)=\bigoplus_{p+q \leq n} V^{p, q}, \quad W_{n}\left(\tilde{V}^{\bullet \bullet}\right)=\widetilde{W_{n}\left(V^{\bullet \bullet}\right)}
$$

the weight filtration of the bigraded space $V^{\boldsymbol{*}^{*}}$ and of the associated bundle. Then each connection in Proposition 2.4.2 preserves $W_{n}\left(\widetilde{V}^{\bullet \bullet}\right)$ and induces the trivial connection on the quotients.

Isomorphisms among connections in Proposition 2.4.2 which induce the identity on the quotients of the weight filtration correspond to gauge transformations

$$
\Omega \mapsto g^{-1} d g+g^{-1} \Omega g, \quad g=g\left(t_{1}, t_{2}\right)=\sum_{p, q \geq 0} C_{p, q} t_{1}^{p} t_{2}^{q}, \quad C_{0,0}=1,
$$


where $C_{p, q}$ is an endomorphism of $V^{\boldsymbol{\bullet}^{\bullet}}$ of bidegree $(-p,-q)$.

Proposition 2.4.4. Each equivalence class of connections as in Proposition 2.4.2 with respect to transformations (2.4.3) contains a unique connection satisfying

$$
A_{p, q}+B_{p, q}=0 \text { for all } p, q \text {. }
$$

The proof is easy, by induction on the length of the weight filtration.

Remark 2.4.5. The condition in Proposition 2.4.4 is a particular case of the so-called Fock-Schwinger gauge condition in physics, which for a connection

$$
\nabla=d+\sum \mathcal{A}_{v}\left(t_{1}, \ldots, t_{n}\right) d t_{v}
$$

in a trivial bundle over $\mathbb{R}^{n}$ or $\mathbb{C}^{n}$ reads:

$$
\sum_{v} t_{v} \mathcal{A}_{v}\left(t_{1}, \ldots, t_{n}\right)=0
$$

2.5. A different set of generators of the Lie algebra $\mathfrak{R}$. We now have two ways of describing a complex mixed Hodge structure $\left(V, W_{\bullet}, F^{\circ}, F^{\prime \prime}\right.$ ) with fixed weight quotients $\operatorname{gr}^{W}(V)=V^{\bullet}$. The first one is by means of the Deligne operator $\delta$ or, equivalently, of the components $D_{p, q}, p, q \geq 1$, of $D=\log (\delta)$. The second one is by means of an equivariant connection, which, by Proposition 2.4.4, we can uniquely represent by the gauge potential

$$
\Omega=\sum_{p, q \geq 1} A_{p, q}\left(t_{1}^{p-1} t_{2}^{q} d t_{1}-t_{1}^{p} t_{2}^{q-1} d t_{2}\right),
$$

where the $A_{p, q}$ satisfy the same homogeneity conditions as the $D_{p, q}$ and, apart from these conditions, can be taken arbitrarily. The connection $\nabla=d+\Omega$ is trivial along the coordinate axes $t_{i}=0$, so Theorem 2.3.1 gives the following relation between the $D_{p, q}$ and the $A_{p, q}$ :

$$
D=\sum_{p, q \geq 1} D_{p, q}=\log P \exp \int_{(-1,0)}^{(0,-1)} \Omega .
$$

Here we used the notation $P \exp \int$ (path-ordered exponential) for the holonomy of a connection. This implies the following.

Proposition 2.5.1. There exist universal relations

$$
D_{p, q}=(-1)^{p+q}\left(\begin{array}{c}
p+q \\
p
\end{array}\right) A_{p, q}+S_{p, q}\left(\left\{A_{p^{\prime}, q^{\prime}} \mid p^{\prime}<p, q^{\prime}<q\right\}\right),
$$

where $S_{p, q}$ are Lie polynomials with rational coefficients in the lower $A_{p^{\prime}, q^{\prime}}$, bihomogeneous of bidegree $(-p,-q)$. 
To prove the proposition, we repeat the above reasoning in the "universal" situation. Consider the free bigraded Lie algebra

$$
\mathfrak{R}^{\prime}=\operatorname{FLie}\left\{\alpha_{p, q} \mid p, q \geq 1, \operatorname{deg}\left(\alpha_{p, q}\right)=(-p,-q)\right\},
$$

similarly to the one in Reformulation 2.2.1, and let $\mathbb{R}_{\leq n}^{\prime}$ be the subalgebra generated by the $\alpha_{p, q}$ with $p+q \leq n$. Let $R_{n}$ be the completed universal enveloping algebra of $\mathfrak{R}_{\leq n}^{\prime}$, i.e., the algebra of noncommutative formal power series in the $\alpha_{p, q}, p+q \leq n$. We then have a connection on $\mathbb{A}^{2}$ with values in $R_{n}$ :

$$
\nabla=d+\omega, \quad \omega=\sum_{p+q \leq n} \alpha_{p, q}\left(t_{1}^{p-1} t_{2}^{q} d t_{1}-t_{1}^{p} t_{2}^{q-1} d t_{2}\right) .
$$

The holonomy of this connection along the segment $[(-1,0),(0,-1)]$ is then a welldefined element of $R_{n}$, and we consider its $\log$ arithm $z$ and the bihomogenous components $z_{p, q}$ of $z$ :

$$
z=\sum_{p, q \leq n} z_{p, q}=\log P \exp \int_{(-1,0)}^{(0,-1)} \omega
$$

Since $z$ is a primitive element, it is a Lie series in the $\alpha_{p, q}, p+q \leq n$. By degree considerations, each $z_{p, q}$ is a Lie polynomial. Now, modulo the commutators, there is no difference between the path ordered exponential and the usual exponential of the integral, so we have

$$
z \equiv \int_{(-1,0)}^{(0,-1)} \omega=\sum_{p+q \leq n} \alpha_{p, q} \int_{-1}^{0}-t^{p-1}(-1-t)^{q-1} d t,
$$

whence the claim.

We can view the variables $z_{p, q}$ as the generators of the free Lie algebra $\mathfrak{R}$ from Reformulation 2.2.1. So taking the logarithm of the holonomy defines a homomorphism

$$
\mathfrak{L} \rightarrow \mathfrak{L}^{\prime}, \quad z_{p, q} \mapsto(-1)^{p+q}\left(\begin{array}{c}
p+q \\
p
\end{array}\right) \alpha_{p, q}+S_{p, q}\left(\left\{\alpha_{p^{\prime}, q^{\prime}} \mid p^{\prime}<p, q^{\prime}<q\right\}\right),
$$

which, because of its triangular form, is an isomorphism. In particular, the $\alpha_{p, q}$ can be expressed back through the $z_{p, q}$ and provide an alternative system of bihomogeneous generators of $\mathfrak{Q}$.

Remark 2.5.2. The generators $\alpha_{p, q}$ essentially coincide with the generators introduced by Goncharov [8] starting from totally different principles. In fact, the main feature in Goncharov's approach to mixed Hodge structures is a connection on the affine line $\mathbb{A}^{1}$ (called the "twistor line" in [8]) whose holonomy along $[0,1]$ is equal to the Deligne operator $\delta$. In our approach, this connection appears as the restriction of a $\mathbb{G}_{m}^{2}$-equivariant connection from $\mathbb{A}^{2}$ to the affine line $t_{1}+t_{2}=-1$. 
2.6. Geometric interpretation of $\mathfrak{R}$. We would now like to give a different interpretation of the bigraded free Lie algebra $\mathfrak{Z}$. Namely, we observe that $\mathfrak{R}$ can be identified with the commutant of the free Lie algebra on two generators.

To be precise, let $A$ be a $\mathbb{C}$-vector space, and let

$$
\operatorname{FLie}(A)=\bigoplus_{d \geq 1} \operatorname{FLie}_{d}(A)
$$

be the free Lie algebra on $A$ graded by the degree of commutator monomials, i.e., by putting $A$ in degree 1 and requiring that the bracket be compatible with the grading. Assume that $A$ is finite-dimensional. Then the algebraic group $\operatorname{GL}(A)$ acts on $\operatorname{FLie}(A)$ by Lie algebra automorphisms. We are interested in the action on the commutant

$$
[\operatorname{FLie}(A), \operatorname{FLie}(A)]=\operatorname{FLie}_{\geq 2}(A) .
$$

This commutant, considered as an abstract Lie algebra, is free. Indeed, by the Shirshov-Witt theorem ([15], Ch. 2) any subalgebra of a free Lie algebra is free. However, there is no canonical choice of free generators for the commutant. For different choices the "spaces of generators" are identified with each other via the identification with the first homology space ("indecomposable elements")

$$
H_{1}^{\mathrm{Lie}}\left(\mathrm{FLie}_{\geq 2}(A), \mathbb{C}\right)=\mathrm{FLie}_{\geq 2}(A) /\left[\mathrm{FLie}_{\geq 2}(A), \mathrm{FLie}_{\geq 2}(A)\right]
$$

In other words, if $B \subset \mathrm{FLie}_{\geq 2}(A)$ is a graded subspace, then the following are equivalent:

(1) The natural map $\operatorname{FLie}(B) \rightarrow \mathrm{FLie}_{\geq 2}(A)$ is an isomorphism, so $B$ is a space of free generators;

(2) The projection $B \rightarrow H_{1}^{\mathrm{Lie}}\left(\mathrm{FLie}_{\geq 2}(A), \mathbb{C}\right)$ is an isomorphism.

The first homology space (2.6.1) was identified, as a GL( $A)$-module, by Reutenauer ([15], (8.6.12)). His result reads:

$$
H_{1}^{\mathrm{Lie}}\left(\mathrm{FLie}_{\geq 2}(A), \mathbb{C}\right)=\bigoplus_{d \geq 1} \Sigma^{d, 1}(A),
$$

where $\Sigma^{d, 1}$ is the Schur functor (irreducible representation of GL) corresponding to the Young diagram $(d, 1)$. It is also well known (see, e.g., [9], Proposition 14.2.2) that as a $\mathrm{GL}(V)$-module,

$$
\bigoplus_{d \geq 1} \Sigma^{d, 1}(A)=\Omega_{\mathrm{pol}}^{2, \mathrm{cl}}\left(A^{*}\right)
$$

is just the space of closed polynomial 2-forms on the affine space $A^{*}$.

Now assume that $A=\mathbb{C}^{2}$ is 2-dimensional, and $t_{1}, t_{2}$ form the standard basis of $A$. We can consider $t_{i}$ as a linear function on $A^{*}$. Then all 2-forms are closed, so a basis of $\Omega_{\mathrm{pol}}^{2, \mathrm{cl}}\left(A^{*}\right)$ is formed by the monomials

$$
w_{p, q}=t_{1}^{p-1} t_{2}^{q-1} d t_{1} d t_{2}, \quad p, q \geq 1 .
$$


If we equip FLie $\left(\mathbb{C}^{2}\right)$ with the bigrading starting with

$$
\operatorname{deg}\left(t_{1}\right)=(-1,0), \quad \operatorname{deg}\left(t_{2}\right)=(0,-1),
$$

and then restrict this bigrading to $\mathrm{FLie}_{\geq 2}\left(\mathbb{C}^{2}\right)$, then $w_{p, q}$ has the same bidegree as the generator $z_{p, q} \in \mathfrak{L}$, namely $(-p,-q)$. So there is a graded isomorphism of Lie algebras

$$
\phi: \mathfrak{L} \rightarrow \mathrm{FLie}_{\geq 2}\left(\mathbb{C}^{2}\right) .
$$

There is, however, a choice in constructing such a $\phi$, which is a choice of lifting of each $w_{p, q}$ to an element $\phi\left(z_{p, q}\right)$ of $\mathrm{FLie}_{\geq 2}\left(\mathbb{C}^{2}\right)$. One possible way to fix these liftings is by putting

$$
\phi\left(z_{p, q}\right)=\left[t_{2},\left[t_{2}, \ldots,\left[t_{2},\left[t_{1},\left[t_{1}, \ldots,\left[t_{1}, t_{2}\right] \ldots\right]=\operatorname{ad}\left(t_{2}\right)^{q-1}\left(\operatorname{ad}\left(t_{1}\right)^{p}\left(t_{2}\right)\right) .\right.\right.\right.\right.
$$

2.7. $\mathfrak{L}$ as a fundamental Lie algebra. It was further shown in ([10], (4.3)), that for any finite-dimensional $A$ as before, the Lie algebra $\mathrm{FLie}_{\geq 2}(A)$ is acted upon not just by $\mathrm{GL}(A)$ but by the group of formal changes of coordinates in $A$, i.e., by

$$
\text { Aut } \mathbb{C}\left[\left[s_{1}, \ldots, s_{n}\right]\right] \quad \text { if } A^{*}=\bigoplus_{i=1}^{n} \mathbb{C} \cdot s_{i} .
$$

This group is pro-unipotent, while $\mathrm{FLie}_{\geq 2}(A)$ is a discrete, infinitely generated Lie algebra. The action, when restricted to any finitely generated subalgebra in $\mathrm{FLie}_{\geq 2}(A)$, factors through a finite-dimensional unipotent quotient.

Alternatively, this means that to any smooth $n$-dimensional algebraic variety $X / \mathbb{C}$ and any point $x \in X$ there is a naturally associated fundamental Lie algebra $\mathcal{P}(X, x)$ which is isomorphic to $\mathrm{FLie}_{\geq 2}\left(\mathbb{C}^{n}\right)$ but not canonically. Any choice of a formal coordinate system near $x$ gives rise to an isomorphism between the two. It was also shown that the first cohomology of $\mathcal{P}(X, x)$ is naturally identified as follows:

$$
H_{\text {Lie }}^{1}(\mathcal{P}(X, x), \mathbb{C})=\hat{\Omega}_{X, x}^{2, \mathrm{cl}} .
$$

Here the right-hand side is the space of formal germs of closed 2-forms on $X$ near $x$.

Further, there is a "nonabelian" version of (2.7.1), which was proved in [10]. Let $\mathrm{g}$ be any complex Lie algebra. Then $H_{\mathrm{Lie}}^{1}(\mathfrak{g}, \mathbb{C})$ is the same as the group of isomorphism classes of 1-dimensional representations of $\mathfrak{g}$, with the operation induced by tensor product. So we denote by $\operatorname{Rep}(\mathfrak{g})$ the tensor category of finite-dimensional representations of $\mathrm{g}$. Then we have an equivalence of tensor categories

$$
\operatorname{Rep}(\mathcal{P}(X, x)) \simeq \widehat{\operatorname{Bun}}_{\nabla}(X, x),
$$

where the right-hand side is the category of formal germs of vector bundles with connections on $X$ near $x$; see [10], Theorem 4.4.3. This equivalence is natural with respect to maps of manifolds preserving base points, in particular, to any algebraic group $G$ acting on $(X, x)$. 
We now specialize to $X=\mathbb{A}^{2}$, with $G=\mathbb{G}_{m}^{2}$ acting diagonally. We get that the category of bigraded representations of $\mathcal{P}\left(\mathbb{A}^{2}, 0\right)=\mathfrak{L}$ is equivalent to the category of $\mathbb{G}_{m}^{2}$-equivariant objects in $\widehat{\operatorname{Bun}}_{\nabla}\left(\mathbb{A}^{2}, 0\right)$. Now, an equivariant formal germ of a connection on $\mathbb{A}^{2}$ near 0 must be a polynomial one by degree reasons. So the latter category is identified with $\operatorname{Bun} \nabla\left(\mathbb{A}^{2} ; \mathbb{G}_{m}^{2}\right)$. This provides an alternative way to relate our description of $\mathrm{MHS}_{\mathbb{C}}$ with Theorem 2.1.5.

\section{References}

[1] A. A. Berlinson, Notes on absolute Hodge cohomology. In Applications of algebraic $K$-theory to algebraic geometry and number theory, Part I, Contemp. Math. 55, Amer. Math. Soc., Providence, RI, 1986, 35-68. Zbl 0621.14011 MR 862628

[2] J. A. Carlson, Extensions of mixed Hodge structures. In Journées de Géometrie Algébrique d'Angers, Juillet 1979, Sijthoff \& Noordhoff, Alphen aan den Rijn 1980, 107-127. Zbl 0471.14003 MR 605338

[3] A. Connes, Trace formula in noncommutative geometry and the zeros of the Riemann zeta function. Selecta Math. (N.S.) 5 (1999), 29-106. Zbl 0945.11015 MR 1694895

[4] A. Connes and M. Marcolli, Renormalization and motivic Galois theory. Internat. Math. Res. Notices 2004 (2004), 4073-4091. Zbl 1131.81021 MR 2109986

[5] P. Deligne, Équations différentielles à points singuliers réguliers. Lecture Notes in Math. 163, Springer-Verlag, Berlin 1970. Zbl 0244.14004 MR 0417174

[6] P. Deligne, Théorie de Hodge, II. Inst. Hautes Études Sci. Publ. Math. 40 (1971), 5-57. Zbl 0219.14007 MR 0498551

[7] P. Deligne, Structures de Hodge mixtes réelles. In Motives (Seattle, WA, 1991), Proc. Sympos. Pure Math. 55, Part 1, Amer. Math. Soc., Providence, RI, 1994, 509-514. Zbl 0824.14005 MR 1265541

[8] A. B. Goncharov, Hodge correlators. Preprint 2008. arXiv:0803.0297

[9] I. M. Gelfand, M. M. Kapranov, and A. V. Zelevinsky, Discriminants, resultants, and multidimensional determinants. Math. Theory Appl., Birkhäuser, Boston 1994. Zbl 0827.14036 MR 1264417

[10] M. Kapranov, Free Lie algebroids and the space of paths. Selecta Math. (N.S.) 13 (2007), 277-319. Zbl 1149.14003 MR 2361096

[11] G. Laumon, Transformation de Fourier homogène. Bull. Soc. Math. France 131 (2003), 527-551. Zbl 1088.11044 MR 2044494

[12] Yu. I. Manin, Gauge field theory and complex geometry. 2nd ed., Grundlehren Math. Wiss. 289, Springer-Verlag, Berlin 1997. Zbl 0884.53002 MR 1632008

[13] O. Penacchio, Structures de Hodge mixtes et faisceaux réflexifs semistables. C. R. Acad. Sci. Paris Sér I Math. 335 (2002), 475-480. Zbl 1030.14009 MR 1937116

[14] O. Penacchio, Structures de Hodge mixtes et fibrés sur le plan projectif complexe. Ph.D. thesis, Université Paul Sabatier, Toulouse, 2002. arXiv:math/0307156

Mixed Hodge structures and equivariant sheaves on the projective plane. Math. Nachr. 284 (2011), 526-542. Zbl 1217.14008 MR 2799248 
[15] C. Reutenauer, Free Lie algebras. London Math. Soc. Monogr. (N.S.) 7, Oxford Science Publications, Oxford 1993. Zbl 0798.17001 MR 1231799

[16] C. Simpson, Mixed twistor structures. Preprint 1997. arXiv:alg-geom/9705006

[17] R. S. Ward and R. O. Wells, Jr., Twistor geometry and field theory. Cambridge Monogr. Math. Phys., Cambridge University Press, Cambridge 1990. Zbl 0714.53059 MR 1054377

Received May 29, 2010

M. Kapranov, Department of Mathematics, Yale University, New Haven, CT 06520, U.S.A.

E-mail: mikhail.kapranov@yale.edu 